\title{
Pathogenicity Stimulation of Sclerotinia sclerotiorum by Subtoxic Doses of Carbendazim
}

Ya-Li Di, Zu-Qing Zhu, Xiao-Ming Lu, and Fu-Xing Zhu, College of Plant Science and Technology, Huazhong Agricultural University, Wuhan, 430070, China

\begin{abstract}
Di, Y.-L., Zhu, Z.-Q., Lu, X.-M., and Zhu, F.-X. 2015. Pathogenicity stimulation of Sclerotinia sclerotiorum by subtoxic doses of carbendazim. Plant Dis. 99:1342-1346.

Sclerotinia sclerotiorum is a devastating ascomycete fungus capable of infecting more than 400 species of plants worldwide. Carbendazim has been a principal fungicide for control of this pathogen and high levels of carbendazim resistance have been reported in eastern China. In this study, stimulatory effect of subtoxic doses of carbendazim on pathogenicity of S. sclerotiorum was investigated. All seven field resistant isolates with EC50 values greater than $1,000 \mu \mathrm{g} / \mathrm{ml}$ exhibited stimulated pathogenicity toward detached leaves of rapeseed at subtoxic concentrations of carbendazim. Detailed studies on pathogenicity of two resistant isolates $\mathrm{AH}-17$ and LJ-86 toward potted rapeseed plants and detached leaves demonstrated that carbendazim at 0.2 to $5 \mu \mathrm{g} / \mathrm{ml}$ could

consistently stimulate significantly higher $(P<0.05)$ pathogenicity than the control. On potted rapeseed plants, the percent stimulations on pathogenicity ranged from 18.8 to $22.0 \%$ for isolate $\mathrm{AH}-17$ and from 15.1 to $23.2 \%$ for isolate LJ-86. On detached leaves of rapeseed, the percent stimulations ranged from 18.7 to $31.29 \%$ for isolate $\mathrm{AH}-17$ and from 16.7 to $24.3 \%$ for isolate LJ-86. Studies on stimulation mechanism indicated that secretion of oxalic acid and tolerance to oxidative stresses $\mathrm{H}_{2} \mathrm{O}_{2}$ and paraquat after exposed to subtoxic doses of carbendazim did not change significantly. These results have profound implications for judicious application of fungicides and sustainable management of fungicide resistance.
\end{abstract}

Sclerotinia sclerotiorum (Lib.) de Bary is a necrotrophic plant pathogen notorious for its broad host range and worldwide distributions. S. sclerotiorum can infect 408 species of plants in 278 genera of 75 families, including many economically important crops and vegetables such as oilseed rape, soybean, peanut, sunflower, cauliflower, lettuce, and carrot (Boland and Hall 1994; Bolton et al. 2006; Purdy 1979). Diseases caused by S. sclerotiorum have traditionally been difficult to control mainly due to inadequacy of pathogenresistant cultivar (Bolton et al. 2006). Application of fungicides is still the mainstay for control of $S$. sclerotiorum on oilseed rape and soybean in China (Wang et al. 2014). The benzimidazole fungicide carbendazim has been extensively used to control S. sclerotiorum for more than three decades in China. Repeated and extensive applications of carbendazim have resulted in emergence of carbendazim resistance in field populations of $S$. sclerotiorum in eastern China (Wang et al. 2014; Zhou et al. 2012). Nevertheless, in most regions of China except eastern provinces, carbendazim is still the principal fungicide for control of $S$. sclerotiorum.

Under field conditions, a small amount of pathogen isolates will inevitably be exposed to subtoxic doses of fungicides because of evaporation and drift of sprayed fungicides and uneven distributions of active ingredients. Recommended application rates on fungicide labels are mainly for sensitive isolates and hence may become subtoxic for resistant isolates (Flores and Garzón 2013). In most studies, researchers are mainly interested in inhibitory action of relatively high doses of fungicides on pathogens and potentially stimulatory effect of subtoxic doses of fungicides has been largely ignored. Stimulatory effect of subtoxic doses of chemicals is a general phenomenon and has been reported across a tremendously wide range of biological systems and chemicals (Calabrese 2013; Calabrese and Blain 2011). In a previous study, we reported stimulatory effect of subtoxic doses of the dicarboximide fungicide dimethachlon on pathogenicity and

Corresponding author: Fu-Xing Zhu, E-mail: zhufuxing@mail.hzau.edu.cn

Accepted for publication 7 April 2015.

http://dx.doi.org/10.1094/PDIS-02-15-0161-RE

(C) 2015 The American Phytopathological Society mycelial growth of S. sclerotiorum (Zhou et al. 2014). Stimulations were also reported on percent germinations of some thiabendazoleresistant Penicillium expansum isolates by low concentrations of thiabendazole (Baraldi et al. 2003), and on mycelial growth of Lyophyllum palustre (Peck) by low concentrations of propamocarb (Landry et al. 2011). The stimulatory effect of subtoxic concentrations of fungicides has been observed not only in plant pathogenic fungi but also in pathogenic oomycetes. Phytophthora undulata could be stimulated by low doses of hymexazol (Kato et al. 1990). Growth and pathogenicity of metalaxyl-resistant $P$. infestans was stimulated by low concentrations of metalaxyl (Zhang et al. 1997). Significant increases in Pythium damping-off of geranium with mefenoxam at $1 \times 10^{-6}$ and $1 \times 10^{-1} \mu \mathrm{g} / \mathrm{ml}$ have been reported (Garzón et al. 2011). Numerous reports of stimulation on plant pathogens indicate that fungicide resistant isolates are probably more prone to be stimulated by subtoxic doses of fungicides. This may be partially due to relatively higher concentrations of fungicide that can elicit stimulatory effect, thus more likely to be observed. Mycelial growth of propamocarb and mefenoxam dual-resistant strains of Pythium aphanidermatum, $P$. irregulare, and $P$. ultimum could be stimulated by propamocarb at $1 \mu \mathrm{g} / \mathrm{ml}$, and in some resistant strains of $P$. aphanidermatum, the stimulations were observed with propamocarb even at 1,000 $\mu \mathrm{g} / \mathrm{ml}$ (Moorman and Kim 2004). Garzón et al. (2011) reported that stimulation of sublethal doses of mefenoxam on mycelial growth of a mefenoxam-resistant isolate of $P$. aphanidermatum was about $10 \%$, whereas the percent stimulation on pathogenicity toward geranium seedlings was as high as $61 \%$. The stimulatory effects of subtoxic doses of fungicides on pathogenicity of plant pathogens are closely related to management of plant diseases and therefore should be given due attention. Otherwise, applications of inappropriate concentrations of fungicides may increase rather than decrease severity of plant diseases. The stimulatory effects of subtoxic doses of fungicide on plant pathogens have profound implications for plant disease management. The stimulatory effects of fungicide may be highly detrimental to agricultural productivity because they are likely beneficial to plant pathogens. Fitness factors such as mycelial growth, spore germination, pathogenicity, and toxin production are likely to be stimulated for the benefit of plant pathogens (Flores and Garzón 2013; Garzón and Flores 2013). When exposed to subtoxic doses of the triazole fungicide prothioconazole, Fusarium graminearum produced 
more mycotoxin deoxynivalenol than the control (Audenaert et al. 2010).

Given the current development of carbendazim resistance in $S$. sclerotiorum and extensive applications of carbendazim for control of this pathogen, it is necessary to study the possible stimulations of carbendazim on pathogenicity of S. sclerotiorum. The specific objectives of this study were (i) to determine stimulatory effects of subtoxic doses of carbendazim on pathogenicity of S. sclerotiorum on detached leaves and potted rapeseed plants, and (ii) to explore possible mechanisms for stimulations on pathogenicity by measuring sensitivity to hydrogen peroxide and paraquat, and production of oxalic acid.

\section{Materials and Methods}

Isolates of plant pathogenic fungus. Field isolates of S. sclerotiorum were collected from diseased rapeseed plants with typical symptoms of Sclerotinia stem rot in Anhui Province of China in 2012. Mature sclerotia were stored at $4^{\circ} \mathrm{C}$ in a refrigerator before being cultured on potato dextrose agar (PDA) media. Determination of sensitivity to carbendazim was carried out according to Wang et al. (2014) and seven resistant isolates with $50 \%$ effective concentration (EC50) values higher than $1,000 \mu \mathrm{g} / \mathrm{ml}$ were assayed for stimulatory effect of carbendazim on pathogenicity.

Fungicide. Technical grade carbendazim $(98.1 \%$ active ingredient, Tian Jin Jin Bei Chemical Co. Ltd.) was dissolved in hydrochloric acid ( $\mathrm{HCl}, 0.1 \mathrm{~mol} / \mathrm{liter})$ to produce an $80,000 \mu \mathrm{g} / \mathrm{ml}$ stock solution. The stock solution was stored at $4{ }^{\circ} \mathrm{C}$ in a refrigerator for no longer than 2 weeks before being serially diluted for experiments.

Stimulatory effects of spraying subtoxic doses of carbendazim on pathogenicity of $S$. sclerotiorum on detached leaves of rapeseed plants. Leaves 9 to $12 \mathrm{~cm}$ in diameter were detached from 4- to 5-week-old rapeseed plants growing in the greenhouse at about

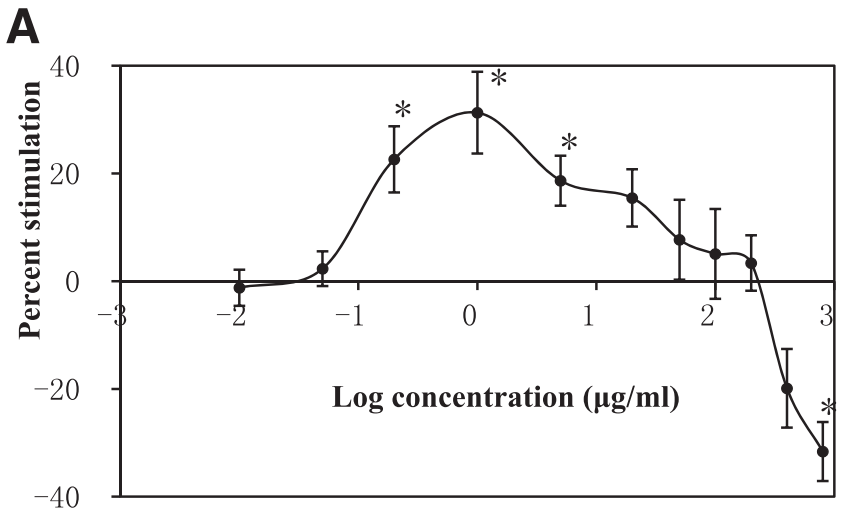

B

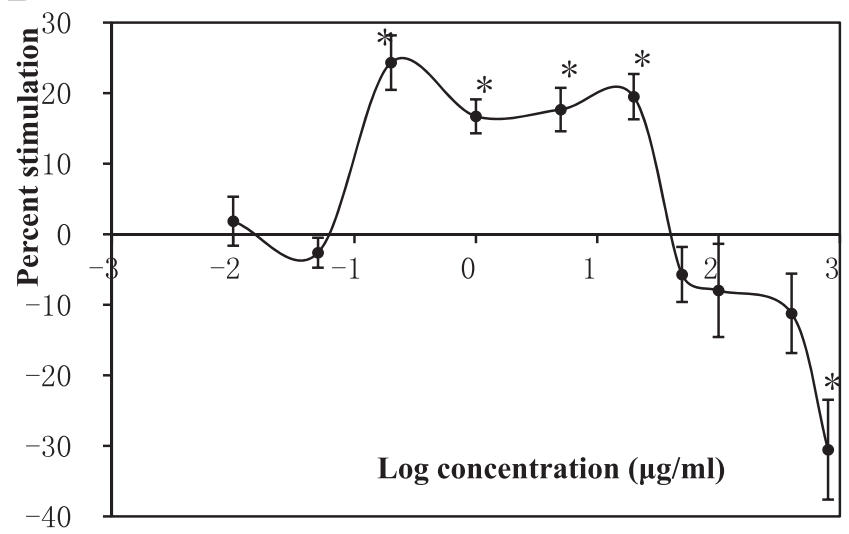

Fig. 1. Stimulatory effect of carbendazim on pathogenicity of isolates $\mathbf{A}, \mathrm{AH}-17$ and $\mathbf{B}$, LJ-86 on detached leaves of oilseed rape plants. Error bar denotes standard error of the mean. Asterisk denotes significant differences from the control. Data are mean values of four repeats. $25^{\circ} \mathrm{C}$. The detached leaves were rinsed with sterile water, air-dried on a clean bench, and transferred to $15-\mathrm{cm}$-diameter petri dishes lined with water-soaked sterilized filter paper to maintain high humidity. Carbendazim solutions were prepared by diluting carbendazim stock solution with $0.1 \mathrm{~mol} / \mathrm{liter} \mathrm{HCl}$ and then further diluted 100 -fold with sterilized $0.1 \%$ Triton- 100 in water to final concentrations of 0.01 , $0.05,0.2,1,5,20,50,100,400$, and $800 \mu \mathrm{g} / \mathrm{ml}$. $\mathrm{HCl}$ at final concentration of $0.001 \mathrm{~mol} / \mathrm{liter}$ in $0.1 \%$ Triton was used as the control. Detached leaves were sprayed with carbendazim dilutions by a handheld sprayer. The treated leaves were air-dried and inoculated on the adaxial surface with inverted mycelial plugs cut from fresh margins of 2-day-old colonies growing on PDA. The inoculated leaves were incubated in a growth chamber at $25^{\circ} \mathrm{C}$, with relative humidity maintained over $85 \%$. Lesion diameters were measured two times at right angles after $48 \mathrm{~h}$ of incubation. The experiment was performed in triplicate and repeated independently four times. All seven resistant isolates exhibited stimulated pathogenicity toward detached leaves of rapeseed at one or more concentrations of carbendazim and isolates AH-17 and LJ-86 were arbitrarily selected for further studies on pathogenicity toward potted rapeseed plants.

Stimulatory effects of spraying subtoxic doses of carbendazim on pathogenicity of $S$. sclerotiorum toward potted rapeseed plants. Carbendazim stock solution was diluted as described above. About 4- to 5-week-old rapeseed plants growing in 20-cm-diameter pots containing a composite mixture of peat and soil (weight ratio, 1:1) were sprayed with the diluted carbendazim solutions. After air drying for about $1 \mathrm{~h}$, leaves were inoculated on the adaxial surface with inverted mycelial plugs. The inoculated plants were incubated in a growth chamber at $25^{\circ} \mathrm{C}$, with relative humidity maintained over $85 \%$. Lesion diameters were measured two times at right angles after $48 \mathrm{~h}$ of incubation. The experiment was performed in triplicate and repeated independently four times.
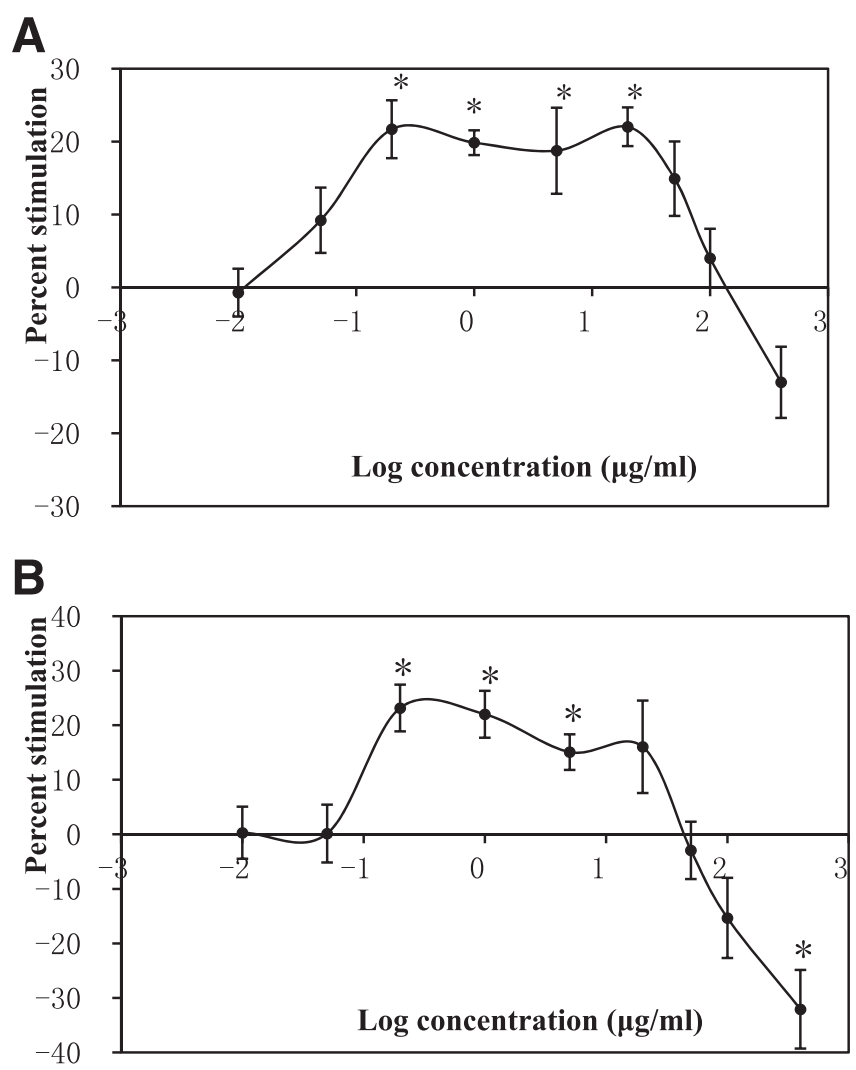

Fig. 2. Stimulatory effect of carbendazim on pathogenicity of isolates $\mathbf{A}, \mathrm{AH}-17$ and $\mathbf{B}$, LJ-86 on potted oilseed rape plants. Error bar denotes standard error of the mean. Asterisk denotes significant differences from the control. Data are mean values of four repeats. 
Effects of subtoxic doses of carbendazim on tolerance of $S$. sclerotiorum to hydrogen peroxide $\left(\mathrm{H}_{2} \mathrm{O}_{2}\right)$ and paraquat. Inverted fresh mycelial plugs were transferred to PDA media amended with carbendazim at 20, 50, 100, and $200 \mu \mathrm{g} / \mathrm{ml}$. PDA amended with $\mathrm{HCl}$ at a final concentration of $2 \times 10^{-3} \mathrm{~mol} / \mathrm{liter}$
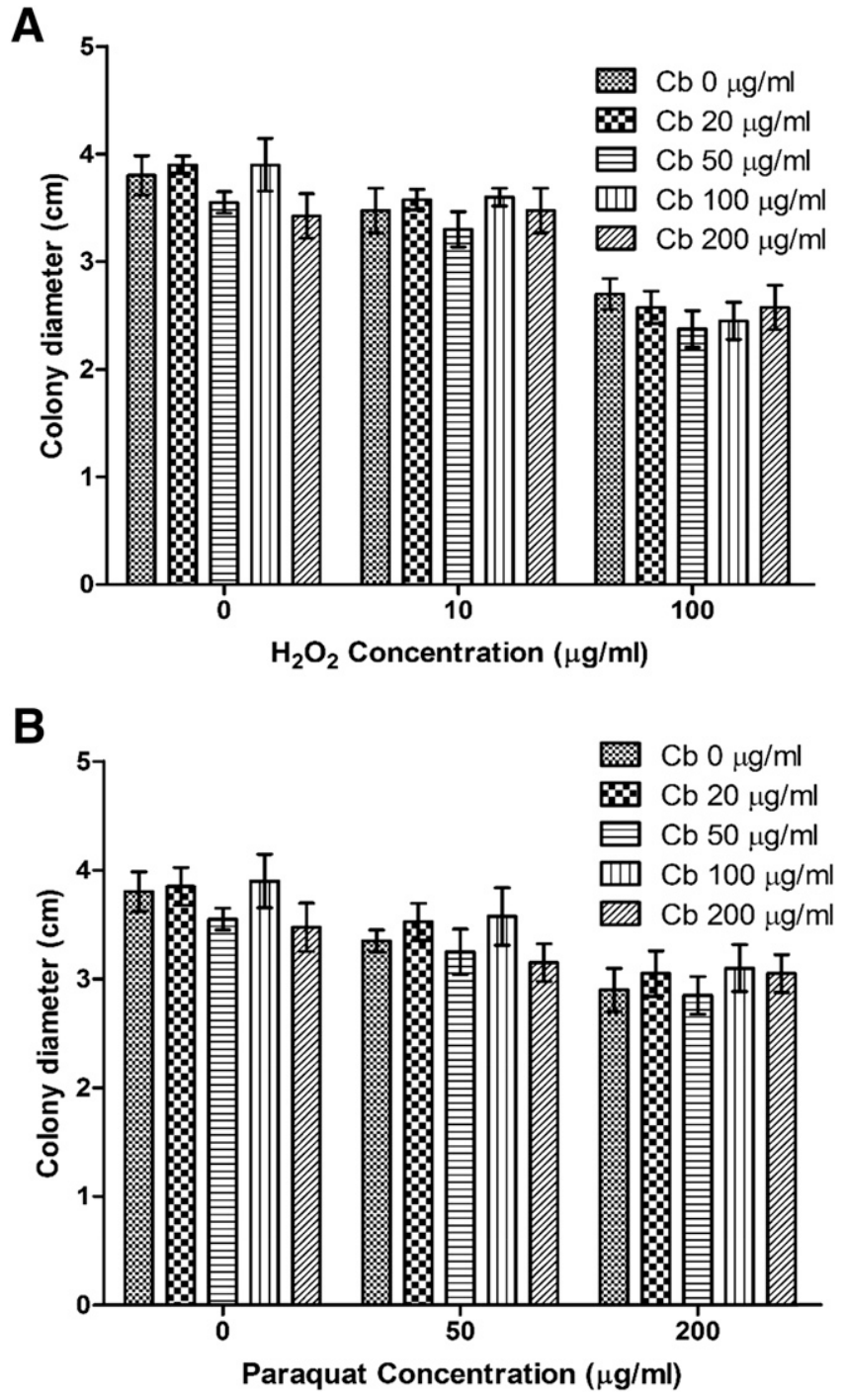

Fig. 3. Effects of pretreatment with carbendazim on sensitivity of isolate LJ-86 to A, $\mathrm{H}_{2} \mathrm{O}_{2}$ and $\mathbf{B}$, paraquat. In this figure, $\mathrm{Cb}$ denotes carbendazim. Each column represents the mean value and standard deviation of six replicates. was used as the fungicide-free control. After incubation in a growth chamber at $23^{\circ} \mathrm{C}$ for $48 \mathrm{~h}$, mycelial plugs cut from fresh margins of these 48-h-old colonies were transferred to PDA media amended with $\mathrm{H}_{2} \mathrm{O}_{2}$ at 10 and $100 \mu \mathrm{g} / \mathrm{ml}$ or with paraquat at 50 and $200 \mu \mathrm{g} / \mathrm{ml}$. After incubating at $23^{\circ} \mathrm{C}$ for $48 \mathrm{~h}$, mycelial growth was calculated by measuring two perpendicular diameters of each colony. The experiment was repeated two times with six replicates in total for each treatment.

Effects of subtoxic doses of carbendazim on oxalic acid production in $\mathbf{S}$. sclerotiorum. Potato dextrose broth (PDB) was amended with carbendazim at final concentrations of $0.2,5,20$, 100 , and $200 \mu \mathrm{g} / \mathrm{ml}$. PDB supplemented with $\mathrm{HCl}$ at a final concentration of $2 \times 10^{-3} \mathrm{~mol} / \mathrm{liter}$ was used as the fungicide-free control. Three mycelial plugs of each isolate were inoculated into $100 \mathrm{ml}$ PDB media amended with carbendazim or $\mathrm{HCl}$ and then incubated at $23^{\circ} \mathrm{C}$ on an orbital shaker at $120 \mathrm{rpm}$. After $72 \mathrm{~h}$ of incubation, PDB media were centrifuged at $12,000 \mathrm{rpm}$ for $10 \mathrm{~min}$ and the supernatants were centrifuged at $12,000 \mathrm{rpm}$ for another $10 \mathrm{~min}$. The supernatants were used to determine oxalic acid concentrations by a colorimetric method according to Duan et al. (2014). Briefly, oxalic acid and iron (III) form a complex that will decrease absorbance at $510 \mathrm{~nm}$ of complex of iron (III) and sulfosalicylic acid under acidic conditions; thus, the content of oxalic acid was calculated from reductions in absorbance at $510 \mathrm{~nm}$ of the complex of iron (III) and sulfosalicylic acid. The standard curve for oxalate was produced as follows: $2 \mathrm{ml} \mathrm{FeCl}$ solution at $0.5 \mathrm{mg} / \mathrm{ml}, 20 \mathrm{ml} \mathrm{HCl}$ $\mathrm{KCl}$ buffer $(\mathrm{KCl} 50 \mathrm{mM}, \mathrm{pH}=2)$, and $1.2 \mathrm{ml}$ sulfosalicylic acid solution at $5 \mathrm{mg} / \mathrm{ml}$ were added into a $50-\mathrm{ml}$ flask. A series of volumes $(0,0.1,0.2,0.4$, and $0.8 \mathrm{ml})$ of sodium oxalate at $2 \mathrm{mg} / \mathrm{ml}$ were then added. The final reaction volume was adjusted to $25 \mathrm{ml}$ by adding double-distilled water. The flasks were vortexstirred and incubated at $25^{\circ} \mathrm{C}$ for $30 \mathrm{~min}$. Absorbance was measured at $510 \mathrm{~nm}$ with an UV-1800 spectrophotometer (SHIMADZU Corporation, Japan). The experiment was performed in triplicate and repeated independently two times.

Data analysis. Percent stimulations were calculated by the following formula. The diameter of mycelial plugs was subtracted from the measured lesion diameters before calculating percent stimulations.

Percent stimulation $(\%)=($ Lesion diameter of the treated - Lesion diameter of the control) / Lesion diameter of the control $\times 100 \%$

Percent stimulations were arcsine square-root transformed before statistical analysis. One-way analysis of variance (ANOVA) and Dunnett's test $(P=0.05)$ in SPSS (Statistical Product and Service Solutions, ver. 19.0, SPSS Inc., Chicago) were used to evaluate whether the percent stimulation was significantly higher than zero. In pot experiments, the experimental unit was defined as each oilseed plant growing in one pot, with the arithmetic mean of lesion diameters as the response. Graphs were created with Microsoft Excel (ver. 2010, Microsoft Corporation, Redmond, WA).
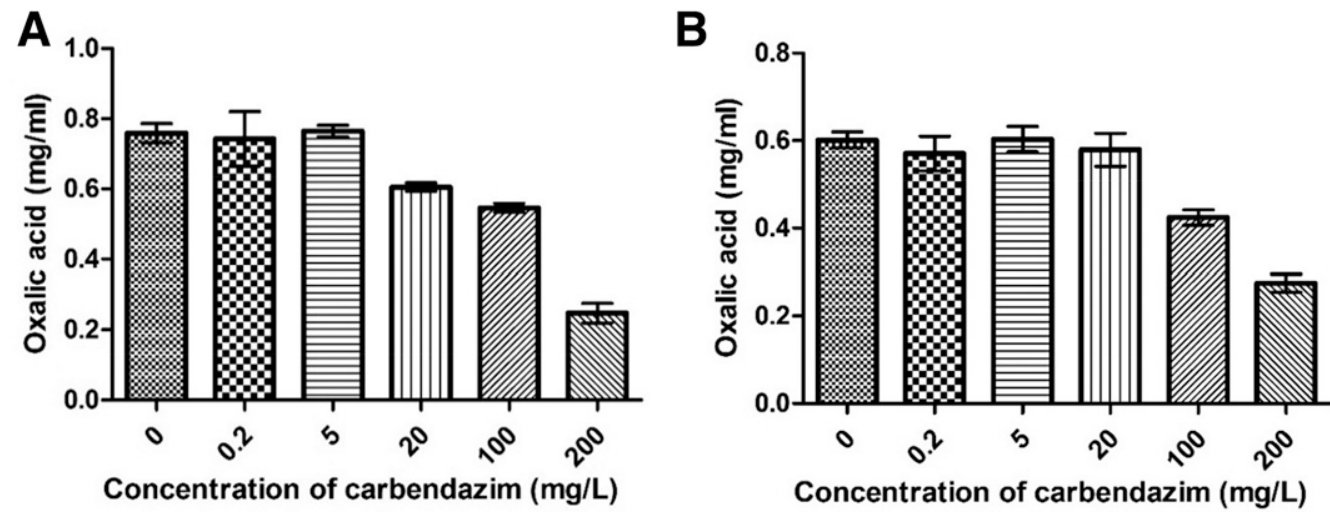

Fig. 4. Oxalic acid production under different concentrations of carbendazim for isolate $\mathbf{A}, \mathrm{AH}-17$ and $\mathbf{B}$, LJ-86. Data are mean values of six replicates. Standard error bar denotes standard error of the mean. 


\section{Results}

Stimulatory effects of spraying subtoxic doses of carbendazim on pathogenicity of $S$. sclerotiorum toward detached leaves of rapeseed. All seven resistant isolates exhibited stimulated pathogenicity toward detached leaves of rapeseed at one or more concentrations of carbendazim. For isolate $\mathrm{AH}-17$, carbendazim at a concentration range from 0.2 to $5 \mu \mathrm{g} / \mathrm{ml}$ had significant $(P<0.05)$ stimulatory effect on pathogenicity and the highest percent stimulation was $31.3 \%$ with carbendazim at $1 \mu \mathrm{g} / \mathrm{ml}$ (Fig. 1). For isolate LJ-86, carbendazim at a range from 0.2 to $20 \mu \mathrm{g} / \mathrm{ml}$ had significant stimulatory effect on pathogenicity. The stimulation profile for isolate LJ-86 was not a typical parabolic curve and the highest stimulation was found with carbendazim at the concentration of $0.2 \mu \mathrm{g} / \mathrm{ml}$.

Stimulatory effects of spraying subtoxic doses of carbendazim on pathogenicity of $S$. sclerotiorum toward potted rapeseed plants. Carbendazim at concentrations from 0.2 to $20 \mu \mathrm{g} / \mathrm{ml}$ showed significant $(P<0.05)$ stimulatory effects on pathogenicity of isolate AH-17 toward potted rapeseed plants (Fig. 2). For isolate LJ-86, the stimulation range was 0.2 to $5 \mu \mathrm{g} / \mathrm{ml}$ and the percent stimulation at $20 \mu \mathrm{g} / \mathrm{ml}$ was not significantly higher than the control mainly due to relatively high standard errors of the mean.

Effects of carbendazim on tolerance of $S$. sclerotiorum to $\mathrm{H}_{2} \mathrm{O}_{2}$ and paraquat. Compared with growth on PDA, isolate LJ-86's growth on PDA amended with $\mathrm{H}_{2} \mathrm{O}_{2}$ at $100 \mu \mathrm{g} / \mathrm{ml}$ was obviously lower (Fig. 3). There was no significant difference $(P=0.1191)$ in mycelial growth on PDA amended with $\mathrm{H}_{2} \mathrm{O}_{2}$ at $100 \mu \mathrm{g} / \mathrm{ml}$ among treatments with different concentrations of carbendazim. Growth on PDA amended with paraquat at $200 \mu \mathrm{g} / \mathrm{ml}$ also showed no significant difference $(P=0.3372)$ among different concentrations of carbendazim. Studies on isolate AH-17 demonstrated similar results (data not shown).

Effects of carbendazim on oxalic acid production in S. sclerotiorum. Carbendazim at final concentrations from 0.2 to $200 \mu \mathrm{g} / \mathrm{ml}$ in PDB did not stimulate oxalic acid production in isolate LJ-86 or AH-17 (Fig. 4). In fact, carbendazim at 20 to $200 \mu \mathrm{g} / \mathrm{ml}$ inhibited production of oxalic acid in isolate $\mathrm{AH}-17$ and the inhibition concentration range for isolate LJ-86 was 100 to $200 \mu \mathrm{g} / \mathrm{ml}$.

\section{Discussion}

Although the stimulation profiles of the present study were not typical hormetic responses, the stimulation magnitude and the stimulatory carbendazim concentration range immediately below the least inhibitory concentration indicated that this stimulatory effect of subtoxic doses of carbendazim to $S$. sclerotiorum was very likely to be the phenomenon hormesis. Hormesis is a toxicological concept about dose-response relationship characterized by a reversal in response between low and high doses of a stressor such as a pesticide (Guedes and Cutler 2014). The concept of hormesis usually refers to a phenomenon of low-dose stimulation and high-dose inhibition (Calabrese 2008; Garzón and Flores 2013); hence, hormetic dose response curves are biphasic rather than monotonic (Hoffmann 2009). In order to detect hormesis scientifically, some experimental design requirements should be met; for instance, evenly distributed doses below the no observed adverse effect level (NOAEL) should be tested to provide enough data for hormesis (Flores and Garzón 2013). Crump (2001) proposed the criteria for evaluating hormesis as follows: strength of evidence, soundness of data, consistency, and biological plausibility. In the present study, at least three concentrations of carbendazim were determined as having significant stimulations on pathogenicity. The dose-response curves of S. sclerotiorum to carbendazim were biphasic.

Hormesis tends to be a prevalent phenomenon that has been observed in a broad range of biological models (from microorganisms and plants to mammals) over numerous and diverse measured endpoints, across a wide range of chemical classes, and independent of mechanisms (Calabrese and Blain 2011). Stimulations on mycelial growth and pathogenicity by subtoxic doses of fungicides have been reported in ascomycetes (Audenaert et al. 2010), basidiomycetes (Flores and Garzón 2013; Landry et al. 2011), and oomycetes (Garzón et al. 2011). Hormesis of insecticide toward arthropod pests and of herbicide toward plants have been recently reviewed by Guedes and Cutler (2014) and Belz and Duke (2014), respectively. The interest in hormesis has recently increased substantially. In the last 20 years, published peer-reviewed papers each year dealing with some aspects of hormesis have increased about 10-fold (Duke 2014).

The stimulations on pathogenicity of $S$. sclerotiorum by subtoxic doses of fungicides have important implications for plant disease management. Some plant pathogens and isolates will inevitably be exposed to subtoxic doses of fungicide due to inappropriate fungicide applications, low-dose applications to reduce costs, and application drift, among other reasons (Flores and Garzón 2013). The stimulatory effects of subtoxic doses of fungicide will potentially result in increased plant disease incidences and severity in the field. In fact, some rapeseed growers have noticed that occasionally, Sclerotinia stem rot caused by $S$. sclerotiorum becomes aggravated after application of carbendazim. Although there is no scientific evidence to substantiate our speculations, it is very likely that the increased Sclerotinia stem rot disease was caused by the stimulatory effects of subtoxic doses of carbendazim, especially for carbendazim-resistant isolates. Therefore, integrated plant disease management should take into account of possible stimulatory effects of subtoxic doses of fungicide on plant pathogens.

The present study demonstrated that pretreatment with subtoxic doses of carbendazim could not enhance tolerance of S. sclerotiorum to oxidative stresses such as $\mathrm{H}_{2} \mathrm{O}_{2}$ or paraquat. Our previous study showed that subtoxic doses of dimethachlon could increase insensitivity to $\mathrm{H}_{2} \mathrm{O}_{2}$ (Zhou et al. 2014). The enhanced tolerance to $\mathrm{H}_{2} \mathrm{O}_{2}$ is very likely one of the reasons for the increased pathogenicity (Williams et al. 2011). The present result demonstrated that mechanism for pathogenicity stimulatory effect of subtoxic doses of carbendazim was not through enhanced tolerance to oxidative stresses. Another possible mechanism for stimulation on pathogenicity was through oxalic acid. Oxalic acid plays an important role in pathogenicity of some Sclerotinia species (Dutton and Evans 1996). Oxalic acid could suppress oxidative burst of host plants (Cessna et al. 2000), enhance activities of cell wall-degrading enzymes by reducing extracellular $\mathrm{pH}$, and had other functions conducive to pathogenicity (Bolton et al. 2006). However, this study demonstrated that subtoxic doses of carbendazim could not stimulate production of oxalic acid. Therefore, the stimulatory effect of subtoxic doses of carbendazim on pathogenicity was not related to oxalic acid. The mechanisms for hormesis are complex (Calabrese 2013). Protecting the organism from injury via adaptive and preconditioning stress responses or overcompensation for a disruption of homeostasis has been claimed to be the mechanistic basis for chemical hormesis (Calabrese et al. 2007). Elucidation of mechanisms for fungicide hormesis will improve our understanding of the nature of the stimulatory effects of subtoxic doses of fungicide on pathogenicity, and thus a better plant disease management strategy may be devised with potential hormetic effects of fungicide being taken into account.

\section{Acknowledgments}

This study was supported by National Natural Science Foundation of China (31371964) and the Special Fund for Agro-scientific Research in the Public Interest (201103016). The authors would like to thank Prof. Daohong Jiang of Huazhong Agricultural University for his invaluable advice.

\section{Literature Cited}

Audenaert, K., Callewaert, E., Höfte, M., Saeger, S. D., and Haesaert, G. 2010 Hydrogen peroxide induced by the fungicide prothioconazole triggers deoxynivalenol (DON) production by Fusarium graminearum. BMC Microbiol. 10:112.

Baraldi, E., Mari, M., Chierici, E., Pondrelli, M., Bertolini, P., and Pratella, G. C. 2003. Studies on thiabendazole resistance of Penicillium expansum of pears: pathogenic fitness and genetic characterization. Plant Pathol. 52:362-370.

Belz, R. G., and Duke, S. O. 2014. Herbicides and plant hormesis. Pest Manag. Sci 70:698-707.

Boland, G. J., and Hall, R. 1994. Index of plant hosts of Sclerotinia sclerotiorum. Can. J. Plant Pathol. 16:93-108.

Bolton, M. C., Thomma, B. P., and Nelson, B. D. 2006. Sclerotinia sclerotiorum (Lib.) de Bary: biology and molecular traits of a cosmopolitan pathogen. Mol. Plant Pathol. 7:1-16. 
Calabrese, E. J. 2008. Hormesis: why it is important to toxicology and toxicologists. Environ. Toxicol. Chem. 27:1451-1474.

Calabrese, E. J. 2013. Hormetic mechanisms. Crit. Rev. Toxicol. 43:580-606.

Calabrese, E. J., Bachmann, K. A., Bailer, A. J., Bolger, P. M., Borak, J., Cai, L., Cedergreen, N., Cherian, M. G., Chiueh, C. C., Clarkson, T. W., Cook, R. R., Diamond, D. M., Doolittle, D. J., Dorato, M. A., Duke, S. O., Feinendegen, L., Gardner, D. E., Hart, R. W., Hastings, K. L., Hayes, A. W., Hoffmann, G. R., Ives, J. A., Jaworowski, Z., Johnson, T. E., Jonas, W. B., Kaminski, N. E., Keller, J. G., Klaunig, J. E., Knudsen, T. B., Kozumbo, W. J., Lettieri, T., Liu, S. Z., Maisseu, A., Maynard, K. I., Masoro, E. J., McClellan, R. O., Mehendale, H. M., Mothersill, C., Newlin, D. B., Nigg, H. N., Oehme, F. W., Phalen, R. F., Philbert, M. A., Rattan, S. I., Riviere, J. E., Rodricks, J., Sapolsky, R. M., Scott, B. R., Seymour, C., Sinclair, D. A., Smith-Sonneborn, J., Snow, E. T., Spear, L., Stevenson, D. E., Thomas, Y., Tubiana, M., Williams, G. M., and Mattson, M. P. 2007. Biological stress response terminology: integrating the concepts of adaptive response and preconditioning stress within a hormetic dose-response framework. Toxicol. Appl. Pharmacol. 222:122-128.

Calabrese, E. J., and Blain, R. B. 2011. The hormesis database: the occurrence of hermetic dose-responses in the toxicological literature. Regul. Toxicol. Pharmacol. 61:73-81.

Cessna, S. G., Sears, V. E., Dickman, M. B., and Low, P. S. 2000. Oxalic acid, a pathogenicity factor for Sclerotinia sclerotiorum, suppresses the oxidative burst of the host plant. Plant Cell 12:2191-2199.

Crump, K. 2001. Evaluating the evidence for hormesis: a statistical perspective. Crit. Rev. Toxicol. 31:669-679

Duan, Y. B., Ge, C. Y., and Zhou, M. G. 2014. Molecular and biochemical characterization of Sclerotinia sclerotiorum laboratory mutants resistant to dicarboximide and phenylpyrrole fungicides. J. Pest Sci. 87:221-230.

Duke, S. O. 2014. Hormesis with pesticides. Pest Manag. Sci. 70:689.

Dutton, M. V., and Evans, C. S. 1996. Oxalate production by fungi: its role in pathogenicity and ecology in the soil environment. Can. J. Microbiol. 42: 881-895.

Flores, F. J., and Garzón, C. D. 2013. Detection and assessment of chemical hormesis on the radial growth in vitro of oomycetes and fungal plant pathogens. Dose Response 11:361-373.
Garzón, C. D., and Flores, F. J. 2013. Hormesis: biphasic dose-responses to fungicides in plant pathogens and their potential threat to agriculture. Pages 311-328 in: Fungicides - showcases of integrated plant disease management from around the world. INTECH. Online: http://cdn.intechopen.com/pdfswm/44734.pdf

Garzón, C. D., Molineros, J. E., Yanez, J. M., Flores, F. J., Jimenez-Gasco, M. M. and Moorman, G. W. 2011. Sublethal doses of mefenoxam enhance Pythium damping-off of geranium. Plant Dis. 95:1233-1238.

Guedes, R. N., and Cutler, G. C. 2014. Insecticide-induced hormesis and arthropod pest management. Pest Manag. Sci. 70:690-697.

Hoffmann, G. R. 2009. A perspective on the scientific, philosophical, and policy dimensions of hormesis. Dose Response 7:1-51

Kato, S., Coe, R., New, L., and Dick, M. 1990. Sensitivities of various oomycetes to hymexazol and metalaxyl. J. Gen. Microbiol. 136:2127-2134.

Landry, J., Martinez, C., and Rochefort, L. 2011. The use of fungicide Nova to mitigate infection of sphagnum by parasitic fungi in the greenhouse. Botany 89:655-661.

Moorman, G. W., and Kim, S. H. 2004. Species of Pythium from greenhouses in Pennsylvania exhibit resistance to propamocarb and mefenoxam. Plant Dis. 88: 630-632.

Purdy, L. H. 1979. Sclerotinia sclerotiorum: history, disease, and symptomatology, host range, geographic distribution, and impact. Phytopathology 69:875-880.

Wang, Y., Hou, Y. P., Chen, C. J., and Zhou, M. G. 2014. Detection of resistance in Sclerotinia sclerotiorum to carbendazim and dimethachlon in Jiangsu Province of China. Australas. Plant Pathol. 43:307-312.

Williams, B., Kabbage, M., Kim, H. J., Britt, R., and Dickman, M. B. 2011 Tipping the balance: Sclerotinia sclerotiorum secreted oxalic acid suppresses host defenses by manipulating the host redox environment. PLoS Pathog 7 : e1002107.

Zhang, S., Panaccione, S. G., and Gallegly, M. E. 1997. Metalaxyl stimulation of growth of isolates of Phytophthora infestans. Mycologia 89:289-292.

Zhou, F., Liang, H. J., Di, Y. L., You, H., and Zhu, F. X. 2014. Stimulatory effects of sublethal doses of dimethachlon on Sclerotinia sclerotiorum. Plant Dis. 98: $1364-1370$

Zhou, F., Wang, Y. F., Zhang, X. L., and Zhu, F. X. 2012. Advances in resistance of Sclerotinia sclerotiorum to carbendazim. Hunan Agric. Sci. 17:82-84. 